\title{
Reliability of electrochemical noise measurements: results of round-robin testing on electrochemical noise
}

Rik-Wouter BOSCH ${ }^{\mathrm{a}}$, Robert A. COTTIS ${ }^{\mathrm{b}},{ }^{*}$, Kinga CSECS $^{\mathrm{c}}$, Thomas DORSCH ${ }^{\mathrm{c}}$, Lucia DUNBAR ${ }^{\mathrm{d}}$, Andreas HEYN ${ }^{\mathrm{e}}$, François HUET ${ }^{\mathrm{f}, 1}$, Outi HYÖKYVIRTA ${ }^{\mathrm{g}}$, Zsolt KERNER ${ }^{\mathrm{h}}$, Alena KOBZOVA ${ }^{\mathrm{i}}$, Jan MACAK ${ }^{\mathrm{j}}$, Radek NOVOTNY ${ }^{\mathrm{k}}$, Johan ÖIJERHOLM ${ }^{1}$, Juha PIIPPO $^{\mathrm{m}}$, Roy RICHNER $^{\mathrm{n}}$, Stefan RITTER ${ }^{\mathrm{o}}$, José M. SÁNCHEZ-AMAYA ${ }^{\mathrm{p}}$, András SOMOGYI $^{\mathrm{q}}$, Saija VÄISÄNEN ${ }^{\mathrm{g}}$, Wenzhong $Z \mathrm{HANG}^{\mathrm{d}}$

a) $S C K \cdot C E N$, Belgium;

b) University of Manchester, School of Materials, P.O. Box 88, Sackville Street, ManchesterM601QD,UK, e-mail: bob.cottis@manchester.ac.uk;

c) AREVA GmbH, Germany;

d) $A M E C, U K$;

e) University of Magdeburg, Germany;

f) UPR15-CNRS, Université Pierre et Marie Curie, France;

g) Technical Research Centre of Finland (VTT), Finland;

h) MTA EK, Centre for Energy Research, Hungary;

i) ÚJV Řež, a.s., Czech Republic;

j) Institute of Chemical Technology, Czech Republic;

k) EC Joint Research Centre Petten, The Netherlands;

l) Studsvik Nuclear AB, Sweden;

m) CORMET Testing Systems, Finland;

n) SIKA Technology AG, Switzerland;

o) Paul Scherrer Institute (PSI), Switzerland;

p) University of Cadiz, Spain.

\begin{abstract}
Sixteen laboratories have performed electrochemical noise (EN) measurements based on two systems. The first uses a series of dummy cells consisting of a "star" arrangement of resistors in order to validate the EN measurement equipment and determine its baseline noise performance, while the second system, based on a previous round-robin in the literature, examines the corrosion of aluminium in three environments. All participants used the same measurement protocol and the data reporting and analysis were performed with automatic procedures to avoid errors. The measurement instruments used in the various laboratories include commercial general-purpose potentiostats and custom-built EN systems. The measurements on dummy cells have demonstrated that few systems are capable of achieving instrument noise levels comparable to the thermal noise
\end{abstract}

\footnotetext{
${ }^{*}$ Corresponding author

${ }^{1}$ ISE member
} 
of the resistors, because of its low level. However, it is of greater concern that some of the instruments exhibited significant artefacts in the measured data, mostly because of the absence of anti-aliasing filters in the equipment or because the way it is used. The measurements on the aluminium samples involve a much higher source noise level during pitting corrosion, and most (though not all) instruments were able to make reliable measurements. However, during passivation, the low level of noise could be measured by very few systems. The round-robin testing has clearly shown that improvements are necessary in the choice of EN measurement equipment and settings and in the way to validate EN data measured. The results emphasise the need to validate measurement systems by using dummy cells and the need to check systematically that the noise of the electrochemical cell to be measured is significantly higher than the instrument noise measured with dummy cells of similar impedance.

Keywords: electrochemical noise, round-robin, corrosion.

\section{Introduction}

Electrochemical noise (EN) measurements are gradually becoming accepted for the study of corrosion processes, although much remains to be done in developing a complete understanding of the interpretation of EN [1]. Furthermore, there is considerable evidence that many EN measurements are contaminated by extraneous noise and measurement artefacts of various sorts. For this reason a round-robin study has been performed to test the quality of EN measurement equipment and to test standard procedures for the validation of such equipments. This paper reports the results of the practical testing; the validation procedure that has been developed has been reported in [2].

This paper does not aim to consider the applications of EN measurements to the study of corrosion processes, nor recent progresses in EN analysis; for this the reader is referred to the extensive literature (e.g. [3]). Instead it focuses on the principles of the measurement process and 
the validation of such measurements. Karel Hladky, one of the pioneers of the application of EN to corrosion, once said "one of the problems with (electrochemical) noise is that it looks pretty much like noise"; more formally, once an EN measurement has been made, it is impossible to distinguish between noise generated by electrochemical processes and that from other sources (there are some exceptions when the extraneous noise has a distinctive character, such as quantisation, but even then the quantisation masks the 'real' EN). It is therefore essential to ensure that the EN measurement system records only EN (at least in as far as is possible with available technology). Attempts have been made in the past to treat this problem; a round-robin experiment was performed by a group based in Germany in the late 1990's [4]. More recently ASTM has developed a standard on EN measurement [5], and there are moves to develop an ISO standard. However, none of these have really provided a definitive indication of the capabilities and limitations of measuring systems that are typically used in modern EN measurements, and for this reason a series of round-robin trials has been undertaken. Each laboratory worked to a common protocol, so the testing conditions were equivalent in all laboratories. The whole point of the exercise was to see how equipment and laboratory usage differences lead to differing results (as is normal for a round-robin).

Three trials have been performed. In the first (RR1), measurements were performed on a series of dummy cells. In that case, the purpose was to have a system in which the theoretical noise level is known. Indeed, no deliberate noise sources were used in the dummy cells, and the metal film resistor technology selected was expected to give essentially theoretical levels of thermal noise (the resistors were purchased as a batch and provided to participants, so any sample to sample variation should be minimal). This measurement provided a very severe test of the instrument noise levels of the measuring systems in the trial. A number of problems were experienced during this trial, which was essentially a learning exercise for the participants.

In the second trial (RR2) the corrosion system first studied by Goellner et al. [4] was used as a test of a real system. However, the results suggested that some of the problems found in RR1 persisted, and in the third round-robin trial (RR3) the experiments of the first two trials were 
combined, together with the use of a detailed set of guidance notes (summarised in [2]) in order to eliminate some of the procedural problems that had arisen in the first two trials.

This paper summarises the results of all of the trials, focusing primarily on problems that were experienced and the limitations of various measurement systems. A total of 11 makes of measurement system, including commercial general-purpose potentiostats from different manufacturers (Gamry: Reference 600, PCI4/300, PCI4/750, ESA400 software; Autolab: PGSTAT 30, GPES software; Solartron: SI 1287, CorrWare software; Jaissle: ZR01/ZU01; ACM: Gill 8 1096; Zahner: IM6, Thales software; IPS: ZRA-FG/HRU-FG, EcmNoise software), and two custom-built EN systems were used in 16 laboratories. The equipment used in each laboratory is not identified for reasons of confidentiality.

\section{Experimental}

Two experimental systems have been used. Both used the conventional three electrode measurement configuration, with current noise measured between two nominally identical working electrodes, and potential noise of the coupled working electrode pair measured relative to a reference electrode. The experimental protocols for the trials are presented in the Appendix in exactly the way that they were presented to participants, since they are a key definition of the round-robin test, i.e. they are necessary for anyone else to reproduce the test.

\subsection{Part 1}

In this trial, performed in RR1 and RR3, three dummy cells were used, each consisting of a 'star' arrangement of three resistors (Fig. 1). The resistors for one dummy cell all had the same value, $100 \Omega$ for cell $\mathrm{A}, 10 \mathrm{k} \Omega$ for cell $\mathrm{B}$ and $1 \mathrm{M} \Omega$ for cell $\mathrm{C}$. All resistors were purchased as a batch, and each batch consisted of a single strip of bandolier mounted devices (see Fig. 2 in [2]), ensuring that they all came from the same production batch. The resistors were $0.75 \mathrm{~W}, 1 \%$ metal 
film devices (Welwyn FR5). Three sampling rates were specified, $1 \mathrm{~Hz}$, the fastest rate that the system was capable of, and an intermediate value (see Appendix).

The dummy cells are expected to produce low levels of current and potential noise, primarily the Johnson (thermal) noise of the resistors. This is known to give white noise (noise with a constant power spectral density, PSD, at all frequencies) with PSDs of $6 k T R$ for the potential noise and $2 k T / R$ for the current noise, where $k$ is Boltzmann's constant, $T$ is the temperature in $\mathrm{K}$ and $R$ is the resistance in Ohms, as derived in Ref. [2]. The measurement of these low levels of noise is difficult, and the primary aims of this trial were to determine the instrument noise levels, and to detect gross errors in the measurement system. A range of resistor values was used because the source impedance is expected to affect the noise levels of both voltage and current amplifiers.

\subsection{Part 2}

In this trial, performed in RR2 and RR3, a 'real' corrosion system was used, aluminium (99.8\% purity) corroding in three solutions, (a) borate buffer solution of $\mathrm{pH} 6.4$, (b) $0.1 \mathrm{M} \mathrm{NaCl}$ solution and (c) $0.5 \mathrm{M} \mathrm{NaNO}_{3}$ solution. The aluminium electrodes were cut from a single rod, and were supplied to participants pre-mounted in epoxy resin. After surface preparation, the electrodes were exposed to the three solutions in turn and the EN monitored (see Appendix). The reference electrode used was silver-silver chloride. The corrosion behaviour of the aluminium in the three solutions was expected to be passive in (a), pitting in (b) and repassivating/passive in (c).

\subsection{Reporting and analysis}

In order to facilitate analysis, and with intention of minimising possibilities for undetected data errors, an XML format (described in [6]) was used for submission of data for analysis. This was supported by a 'schema' that could be used to validate aspects of the data file (such as ensuring that numeric fields contained only numbers and that required fields were present). Participants were requested to validate files before submitting, but not everyone was able to do this. 
Analysis was largely automated: firstly the XML files were converted to a Matlab M-file format using an XSLT script running on the Microsoft msxsl.exe program (available at [7]). Some manual editing of the XML files was necessary to modify text that prevented the conversion to valid M-files (notably single quotes in text fields, which Matlab interpreted as an end of string marker). Data errors (such as sign errors for potential measurements) were not corrected. The M-files were then executed within a Matlab program that analysed the data and produced a variety of graphs, including individual and composite current and potential time records and current and potential power spectra. Note that in principle Matlab can interpret XML files directly, but the method that it uses is excessively slow for files with many elements.

The optimum method for the estimation of power spectra remains somewhat controversial, but as many consider Fourier transform methods to be definitive, a variant of the Welch method has been used, in which segments of the time record are transformed to power spectra and the results averaged to reduce the scatter inherent in a Fourier transform of a noise signal [2]. The averaging was necessary for this application, where it was required to plot summary graphs with many spectra on a single graph, as the result of using a single Fourier transform for each time record would have been a mass of solid colour at high frequencies due to the overlapping lines. Depending on the measurement system and the sampling frequency $f_{\text {s, }}$ time records of variable $N$ number of data points were acquired by the participants. For $M$ data points in each individual segment of the time record, the Fourier transform gives $M / 2$ frequency bins, ranging from the Nyquist frequency, $f_{\mathrm{s}} / 2$, down to $f_{\mathrm{s}} / M$, with a uniform bin spacing in the linear frequency space. In the PSD calculation, a low value of $M$ was used $(M=512$ in Part 1 and $M=128$ in Part 2) for all data sets so that the highest possible number of individual segments in the time record was used (at least 4 in Part 2 and 8 in Part 1). Before using the Pwelch function available in Matlab, a linear trend removal for the individual segments of the time record was performed, since this was found to be necessary to avoid low frequency artefacts in the resultant average spectra. 
In the present manuscript, all curves (time records and PSDs) measured with a given equipment in a given laboratory have been plotted with the same colour.

\section{Results}

Owing to the large number of results, only summary graphs are presented here, together with a small selection of individual results to illustrate 'features' observed. Moreover, the summary graphs showing time records and PSDs for the dummy cell $\mathrm{B}(R=10 \mathrm{k} \Omega)$ are not presented since the results are very similar to those obtained with cells $\mathrm{A}(R=100 \Omega)$ and $\mathrm{C}(R=1 \mathrm{M} \Omega)$. In the same way, for Part 2, only the summary graphs for solutions (a) (passive system) and (b) (pitting corrosion) are given since the results for solution (c) (repassivating/ passive system) are similar to those measured with solution (a).

\subsection{Part 1}

This trial is expected to produce current and potential time records with a mean of zero. Deviations of the mean from zero indicate offset errors in the measuring instrument or, in some cases, may be associated with the application of real currents to the test cell, and may, therefore, perturb the system being measured. These ranged from $10^{-13}$ to $2.5 \times 10^{-6} \mathrm{~A}$ and $10^{-5}$ to $3 \times 10^{-3} \mathrm{~V}$.

Fig. 2 shows the summary potential time records sampled at $f_{\mathrm{s}}=1 \mathrm{~Hz}$ ( $2 \mathrm{~Hz}$ for 3 curves) for the $100 \Omega$ and $1 \mathrm{M} \Omega$ dummy cells in RR1 and RR3 (the Part 1 systems were not used in RR2) and Fig. 3 gives the corresponding current time records sampled at the same time. For a clearer reading, the DC levels of the time records have been slightly shifted in both figures. It must be noticed that only time records sampled at the same frequency can be compared since those sampled at high frequencies, typically $1 \mathrm{kHz}$, contain more frequencies and therefore have a larger amplitude. It can be seen that the plots are dominated by a few very high amplitude results. Results for other resistor

values displayed very similar trends, implying that the results from some laboratories are 
completely dominated by instrument noise. In order that the more typical time records can be seen, results with a range greater than $5 \mathrm{mV}$ have been removed from the summaries in Fig. 2 .

Table 1 gives the highest and lowest values of the standard deviations of the time records shown in Figs. 2 and 3. Theoretical values are also given; they are calculated on the assumption that the potential and current thermal noise signals are white (PSD independent of frequency) down to $1 \mathrm{mHz}$. In that case, the variance of the signal is equal to the PSD multiplied by the maximum frequency analyzed $\left(f_{s} / 2\right)$, so that the standard deviations of the potential and current thermal noise signals are, respectively, $\sqrt{3 k T R f_{S}}$ and $\sqrt{k T f_{S} / R}$. These values give the minimum level of noise that can be measured. Table 1 confirms the large scatter in the noise levels identified in Figs. 2 and 3 , the origin of which will be discussed after the frequency domain analysis. Moreover, the standard deviation values are far above the theoretical levels, which is reasonable because the amplitude of the thermal noise of resistors is very low. However, one instrument in RR1 gave standard deviations values below the theoretical values for the three dummy cells, probably because of an error in the gain of the current channel of the instrument.

To confirm the validity of these noise measurements, a frequency analysis must be performed from time records sampled at various frequencies [2]. Figs. 4 and 5 show summary potential and current power spectra for all dummy cell trials (Part 1) in RR1 and RR3. For the PSD calculation, between 8 and 58 segments of $M=512$ data points were used depending of the number $N$ of points acquired. As indicated above, all potential and current PSDs with the same colour have been measured with the same instrument in the same laboratory. The theoretical noise level due to the thermal noise of the resistors is indicated on the power spectra by a horizontal dashed line. Because of the difficulty of measuring the low levels of the thermal noise produced by the dummy cell, it is not expected that instruments will measure the theoretical noise levels at all (or any) frequencies. However, some systems do appear to be able to measure the thermal voltage and current noises at higher frequencies on the $1 \mathrm{M} \Omega$ dummy cell (Figs. $4 \mathrm{~d}$ and $5 \mathrm{~d}$ ). Note that all spectra display a rising PSD with falling frequency at lower frequencies. The latter may be 
attributable to low frequency $1 / f$ noise [8]. The large scatter in the values of standard deviation in Table 1 is confirmed by PSD levels at a given frequency $(0.5 \mathrm{~Hz})$ ranging inside about 7 decades for the potential and 10 decades for the current, for both RR1 and RR3. This is a strong indication that most commercial equipment, or the inadequate way they are used, do not permit valid noise measurements. Another indication of invalid measurements is revealed by the fact that PSDs for the same instrument measured at several sampling frequencies do not overlap well in the common frequency range analysed because of the absence or misuse of anti-aliasing filters in the data acquisition systems before the analogue-to-digital conversion. Curiously, some examples may be found where different users working with the same commercial equipment did not measure the same level of noise on the dummy cells. This is probably due to the fact that the choice of the voltage and current ranges has a significant impact on the level of the instrumentation noise: the measurement of low-level noise implies using the highest gains of the voltage and current channels consistent with avoiding overloading.

Moreover, these spectra reveal a number of problems:

(i) $A C$ interference. Several of the measurements made at higher frequencies (above $50 \mathrm{~Hz}$ ) display AC interference, visible on the time records as a broad band of data of relatively constant amplitude, often with a ripple in the peak amplitude due to beating between the sampling frequency and the power line frequency, as in Fig. 6a. This results in peaks in the power spectra, with the fundamental at $50 \mathrm{~Hz}$ and harmonics at multiples of $50 \mathrm{~Hz}$ (Fig. 6b). This may not be a problem with the measuring equipment, but an indication that the laboratory concerned did not provide very good shielding of the measurement system. However, some systems display similar peaks at lower frequencies when sampling frequencies below $50 \mathrm{~Hz}$ were used (peak at $4.5 \mathrm{~Hz}$ in Fig. 6c), which indicates that the measuring system did not provide appropriate anti-aliasing filtering able to remove the influence of $50 \mathrm{~Hz}$ and harmonics. This was confirmed by the absence of the drop in PSD at frequencies just below $f_{\mathrm{s}} / 2$ that is indicative of the presence of anti-aliasing filters. For comparison a system with good anti-aliasing filters displays mains frequency peaks when sampled 
at high frequency, but they are absent when the sampling frequency is $1 \mathrm{~Hz}$ : The left spectrum in Fig. $6 \mathrm{~b}$ shows the absence of aliased mains frequency and the drop in PSD due to the effect of the anti-aliasing filter.

(ii) Unsuitable filtering. Some spectra also display the results of unsuitable low pass filtering, as in Fig. $6 \mathrm{~d}$ in which the plateau at higher frequencies is due to the instrumentation noise of the filter. Note that there is still a peak at $50 \mathrm{~Hz}$, implying that the filter has a relatively low roll-off slope and has not completely removed the $50 \mathrm{~Hz}$ interference. This unsuitable low pass filtering was usually observed when using commercial instruments with a limited selection of low pass filters, and is arguably a misuse of the instrument by using an inappropriate sampling frequency (in order to meet the requirements of the round-robin), rather than a real problem. A rather puzzling observation in RR1 was that several power spectra dropped well below the theoretical noise level of the resistors. In some cases this was probably due to unsuitable low pass filtering, and possibly in other cases to scaling errors in the original data (although this seems unlikely as the intended scaling was in A and $\mathrm{V}$, so the low numerical values imply scaling in units such as $\mathrm{kA}$ or $\mathrm{kV}$ ). This problem did not recur in RR3, except for some current spectra where filtering of the current-measuring resistor by parasitic capacitance was involved.

(iii) Quantisation. Several time records also display signs of quantisation (e.g. Fig. 7a), particularly in the potential noise. This will be reflected in the power spectra as a higher flat PSD over the entire frequency range (Figs. $7 \mathrm{~b}$ and $4 \mathrm{a}$ ). In general the results in RR3 were better than for RR1, although a few systems still display problems with aliasing and high general noise levels, and they do not appear to be suitable for EN measurements.

\subsection{Part 2}

For the Part 2 measurements the current noise was expected to have a mean of zero, but the potential noise should have a negative mean, since the measurements were expected to include the DC potential relative to the silver-silver chloride $(\mathrm{Ag} / \mathrm{AgCl})$ reference electrode. In practice, some 
time records had a mean of zero, as illustrated in Fig. 8 for section (a) of the experiment, presumably because the mean potential had been subtracted at some stage or a high pass filter had been used before data conversion, and in other cases the mean was positive, indicating that the sign of potential was incorrect. The latter error is important, as the sign of potential fluctuations can be important for confirming that they are caused by pitting or similar events (which should produce a negative-going transient as the current from the pit polarises the cathodic reaction on the surface outside the pit). In most cases it should be obvious if the sign of the DC potential is wrong, unless $E_{\text {corr }}$ happens to be close to zero, or a third electrode of the test material is used as a pseudoreference, as is common in plant applications. The removal of the mean could disguise a sign error, and in this case it would not be possible to tell from the data that this had occurred. For this reason it is best not to remove the mean, or to report the value of any DC offset used to permit a higher amplifier gain.

The large potential range in Fig. 8 makes it impossible to detect the fluctuations in individual time records, so the data are replotted in Fig. 9 after subtraction of a constant potential value close to the mean for a clearer reading of all time records in the same figure. This was done as well in Fig. 10 for the current time records, with the same colours as in Fig. 9 for the corresponding (same equipment in same laboratory) potential time records. A new feature that was not observed in Part 1 arises with real electrochemical systems because of their non-stationarity that leads to drifting potential and current signals, especially when the noise level is very low (sections (a) and (c)). The drifting signals are even at the origin of serious artefacts in a few systems, as shown in Fig. 11 in which the potential shows clear steps of nearly $4 \mathrm{mV}$ (the steps in current are less obvious, but can still be detected). This effect appears to be due to an autozero process or something similar being employed every 200 s. Autoranging will give similar, but less regular, steps due to gain inaccuracies and slight differences in zero setting between ranges, and for this reason it should be avoided [2]. These artefacts were not detected in Part 1, presumably because the instrument works correctly with 
simple resistive inputs. Sign errors may also be observed in a potential time record measured during pitting corrosion (Fig. 9c).

Figs. 12 and 13 show the potential and current power spectra, respectively. Surprisingly, the scatter in the potential PSD levels is larger for pitting corrosion ( 8 decades at $0.5 \mathrm{~Hz}$ ) than for the passive system (4 decades in section (a), 5 decades in section (c)) in RR2. The opposite was found in RR3 (5 decades for the passive system in sections (a) and (c) against 2 decades for pitting corrosion), which is more reasonable since the scatter should be greater for the measurement of low potential noise levels. The scatter is greater in the current PSD, even for the corroding system in RR2 (10, 9, and 7 decades at $0.5 \mathrm{~Hz}$ for sections (a), (b), and (c), respectively). In RR3, the scatter is less for the corroding system ( 2 decades), but the scatter is still large for the passive systems ( 7 decades in both sections (a) and (c)).

A fundamental limitation of the Part 2 experiment is that, unlike Part 1, there is no way of determining the 'correct' result. In RR2 the range of results obtained was very large. In RR3 the range was narrower, presumably thanks to improved protocols, although the range of results was still rather large for sections (a) and (c) where the system was passive. For section (b), the noise is clearly due to pitting corrosion since the current and potential PSDs at $0.5 \mathrm{~Hz}$ (between $10^{-15}$ $10^{-13} \mathrm{~A}^{2} \mathrm{~Hz}^{-1}$ and $10^{-7}-10^{-5} \mathrm{~V}^{2} \mathrm{~Hz}^{-1}$, respectively) are higher than the highest PSD levels measured for the dummy cells in RR3. For sections (a) and (c), it is difficult to identify the PSDs actually measuring the noise of the passive system. In both sections, the higher current PSDs at $0.5 \mathrm{~Hz}$ are of the order of $10^{-15} \mathrm{~A}^{2} \mathrm{~Hz}^{-1}$, and comparison of these data with the Part 1 measurements for similar cell impedances reveal comparable results. Another difficulty of the PSD analysis comes from the fact that the noise measurements have been performed at a single sampling frequency, as asked in the protocol, and it is not possible to know whether PSDs measured at higher sampling frequency would overlap those measured at a lower frequency; some PSDs, especially those flat up to $f_{\mathrm{s}} / 2$, are corrupted by spectrum aliasing. It seems probable that the high amplitude results are largely due to instrument noise. The situation is somewhat better for potential noise, with only a few results 
displaying unusually high PSDs; again these are comparable to the PSDs measured on the dummy cells. These results emphasise the importance of determining the instrument noise performance of any system used to measure EN, especially when used on low-noise systems such as passive alloys. If the PSD observed during a real measurement is comparable to that obtained on a dummy cell, the data must be discarded.

\section{Conclusions}

The laboratories participating in this round-robin exercise all had some experience in EN measurements, and the results reveal the difficulty of achieving reliable EN measurements on lownoise, high-impedance systems such as passive alloys. In RR3, where both Parts 1 and 2 were essentially second attempts, reasonably consistent results were obtained for Part 2 section (b), which exhibits relatively high noise amplitudes, but a much wider range of results was obtained for sections (a) and (c). Since all participants used the same measurement protocol, the large scatter in the data with resistive dummy cells can only be explained by differences in the equipment used (in particular, the absence of anti-aliasing filters leads to bad EN data) and how they are used (for example, autoranging affects the EN measured). On the other hand, looking at the power spectra obtained on dummy cells, it can be seen that almost all of the measurement systems studied have instrument noise levels that could be affecting the measurements, so few of the results in this work can be regarded as reliable. The measurement systems studied include many commercial instruments, and the results emphasise the importance of determining the instrument noise level of the measurement system, and discarding any measurements that do not display a significantly greater PSD than the instrument noise of dummy cells of comparable impedance. It is also important to sample the EN signals with at least two sampling frequencies to control the overlap of the power spectra in common frequency ranges and validate the measured data. This validation process has been detailed in Ref. [2]. 


\section{Acknowledgement}

The support from the members of the European Cooperative Group on Corrosion Monitoring of Nuclear Materials (ECG-COMON) is gratefully acknowledged.

\section{References}

[1] R.A. Cottis, Corrosion, 57 (2001) 265.

[2] S. Ritter, F. Huet, R.A. Cottis, Mater. Corros., 63 (2012) 297.

[3] F. Huet, "Electrochemical noise technique", in: P. Marcus, F. Mansfeld (Eds.) Analytical methods in corrosion science and engineering, Vol. 22, CRC Press, Taylor \& Francis Group, Boca Raton, FL, USA, 2006, p. 507.

[4] J. Goellner, A. Burkert, A. Heyn, J. Hickling, Corrosion, 55 (1999) 476.

[5] ASTM G199-09: Standard guide for electrochemical noise measurement, in: Annual book of ASTM standards, Vol. 03.02, ASTM International, West Conshohocken, PA, USA, 2009.

[6] R.A. Cottis, "An XML format for electrochemical noise data", in: Proceedings of EUROCORR 2006, EFC, Maastricht, The Netherlands, 2006.

[7] http://www.ecg-comon.org/guideline/XSLTscript.html (viewed on September 30, 2013).

[8] D.A. Eden, "Electrochemical noise - the first two octaves", in: Proceedings of CORROSION 1998, Paper No. 386, NACE International, Houston, TX, USA, 1998. 


\section{Appendix A. Protocol used for the electrochemical noise measurements in the round-robin tests}

\section{A.1. Part 1: Electrochemical noise measurements with a dummy cell}

Each dummy cell should be connected to the measuring system in exactly the same way as for a standard measurement. If a Faraday cage is used for normal measurements the dummy cell should be placed in the Faraday cage. Be careful to avoid contacting any part of the dummy cell with conducting surfaces.

Switch the instruments on and allow the system temperature to stabilise for the greater of fifteen minutes or the time you normally allow. All measurements should be made at the maximum sensitivity of the instrument (except in the unlikely event that this leads to overloading) and with other signal conditioning (anti-aliasing filters, etc.) as recommended by the manufacturer or at the values you consider most appropriate or would normally use. Avoid using the automatic setting of the measurement parameters (sensitivity, gain, offset correction, etc.), such as "autorange", or use it once to know the range selected by the instrument, then deselect "autorange" and use this range for the EN measurements.

The testing protocol was the following:

1) Connect the dummy cell.

2) Warm up the system $(t>15 \mathrm{~min})$.

3) Electrochemical noise measurements (with the $100 \Omega, 10 \mathrm{k} \Omega$ and $1 \mathrm{M} \Omega$ dummy cell):

a) EN measurement with the highest possible sampling rate; duration of the measurement depends on the sampling rate: at least 16384 samples (e.g., at $100 \mathrm{~Hz}$ sampling rate, $170 \mathrm{~s}$ measurement time is sufficient).

b) EN measurement for (at least) $1 \mathrm{~h} 10 \mathrm{~min}$ with a sampling rate of $1 \mathrm{~Hz}$ (or as close as possible) to acquire 4096 samples. 
c) EN measurement with a sampling rate between the other two; duration of the measurement depends on the sampling rate: at least 16384 samples (e.g., at $10 \mathrm{~Hz}$ sampling rate, $28 \mathrm{~min}$ measurement time is sufficient).

4) Convert the data into XML files according to the descriptions in [5].

\section{A.2. Part 2: Electrochemical noise measurements with a 'real' corrosion system}

This project assumes the conventional three electrode EN measurement setup, in which the current noise (ECN) between two identical working electrodes (WEs) is measured with a zero resistance ammeter (ZRA). The potential noise (EPN) of this working electrode pair is then measured with respect to a third reference electrode (RE). The ECN and EPN will be recorded before, during and after (metastable) pitting corrosion of aluminium coupon specimens (buffer $\rightarrow$ $\mathrm{NaCl} \rightarrow$ inhibitor).

The testing parameters were as follows:

- WE 1: Al coupon $\left(\mathrm{Al} 99.8, \mathrm{~A}=0.9 \mathrm{~cm}^{2}\right)$

- WE 2: Al coupon $\left(\mathrm{Al} 99.8, \mathrm{~A}=0.9 \mathrm{~cm}^{2}\right)$

- RE: Ag/AgCl-RE

- Solution a) borate buffer, $\mathrm{pH}=6.4$ (passive/no pitting) at room temperature

- Solution b) $0.1 \mathrm{M} \mathrm{NaCl}$ (metastable/stable pitting) at room temperature

- Solution c) $0.5 \mathrm{M} \mathrm{NaNO}_{3}$ (inhibition/no pitting) at room temperature

- EN measurements: EPN and ECN measurement, sampling rate: $1 \mathrm{~Hz}$ (or as close as possible), 10 min measurement period for each phase

The testing protocol was the following:

1) Preparation of the test solutions (please note the higher initial concentrations of the solutions; only after putting them together you will get the desired concentrations):

a) borate buffer solution $(\mathrm{pH}=6.4)$ : $3.81 \mathrm{~g} \mathrm{Na}_{2} \mathrm{~B}_{4} \mathrm{O}_{7}\left(10 \mathrm{H}_{2} \mathrm{O}\right)$ and $30.92 \mathrm{~g} \mathrm{H}_{3} \mathrm{BO}_{3}$ in 11 deionised water. 
b) $1 \mathrm{M} \mathrm{NaCl}$ solution: $58.44 \mathrm{~g} \mathrm{NaCl}$ in 11 deionised water.

c) $4 \mathrm{M} \mathrm{NaNO}_{3}$ solution: $340 \mathrm{~g} \mathrm{NaNO}_{3}$ in 11 deionised water.

2) Fill beaker with solution (a) (e.g., $405 \mathrm{ml}$ ) and prepare the RE-setup.

3) Preparation of the specimens (WE 1 and 2): grind specimens wet with P600 (FEPA) or 400 (ANSI/CAMI) paper (25 $\mu \mathrm{m}$ grain size); rinse with deionised water and alcohol; dry specimens.

4) Immerse specimens into solution (a) immediately after preparation of the specimens.

5) Start EN measurement 50 min after immersion of the specimens: ECN and EPN measurement with a sampling rate of $1 \mathrm{~Hz}$ (or as close as possible to $1 \mathrm{~Hz}$; take care of proper filter settings/aliasing).

6) Stop EN measurement after 10 min.

7) Add solution (b) (e.g., $45 \mathrm{ml})$.

8) Start EN measurement 4 min after addition of solution (b).

9) Stop EN measurement after 10 min.

10) Add solution (c) (e.g., $64.3 \mathrm{ml})$.

11) Start EN measurement 4 min after addition of solution (c).

12) Stop EN measurement after 10 min.

13) Create 3 XML-files according to the instructions given in [6]. 


\section{Tables}

Table 1. Standard deviations of potential $\left(\sigma_{V}\right)$ and current $\left(\sigma_{I}\right)$ time records sampled at $1 \mathrm{~Hz}$ (a few at $2 \mathrm{~Hz}$ ) in RR1 and RR3: theoretical, highest and lowest values.

\begin{tabular}{llllllll} 
& $R$ & $\sigma_{V, \text { theo }}$ & $\sigma_{V, \text { high }}$ & $\sigma_{V, \text { low }}$ & $\sigma_{\text {I, theo }}$ & $\sigma_{\text {I, high }}$ & $\sigma_{\text {I,low }}$ \\
\hline \multirow{2}{*}{$100 \Omega$} & RR1 & $1.1 \mathrm{nV}$ & $370 \mu \mathrm{V}$ & $830 \mathrm{nV}$ & $6.3 \mathrm{pA}$ & $26 \mathrm{nA}$ & $1.4 \mathrm{pA}$ \\
& $\mathrm{RR} 3$ & $1.1 \mathrm{nV}$ & $72 \mu \mathrm{V}$ & $150 \mathrm{nV}$ & $6.3 \mathrm{pA}$ & $48 \mathrm{nA}$ & $21 \mathrm{pA}$ \\
$10 \mathrm{k} \Omega$ & $\mathrm{RR} 1$ & $11 \mathrm{nV}$ & $310 \mu \mathrm{V}$ & $800 \mathrm{nV}$ & $0.63 \mathrm{pA}$ & $430 \mathrm{pA}$ & $0.020 \mathrm{pA}$ \\
& $\mathrm{RR} 3$ & $11 \mathrm{nV}$ & $130 \mu \mathrm{V}$ & $270 \mathrm{nV}$ & $0.63 \mathrm{pA}$ & $89 \mathrm{nA}$ & $11 \mathrm{pA}$ \\
$1 \mathrm{M} \Omega$ & $\mathrm{RR} 1$ & $0.11 \mu \mathrm{V}$ & $34 \mu \mathrm{V}$ & $0.17 \mu \mathrm{V}$ & $0.063 \mathrm{pA}$ & $47 \mathrm{pA}$ & $0.012 \mathrm{pA}$ \\
& $\mathrm{RR} 3$ & $0.11 \mu \mathrm{V}$ & $76 \mu \mathrm{V}$ & $0.54 \mu \mathrm{V}$ & $0.063 \mathrm{pA}$ & $47 \mathrm{nA}$ & $0.53 \mathrm{pA}$
\end{tabular}




\section{Figure captions}

Fig. 1. Dummy cell for Part 1 (all resistors equal value).

Fig. 2. Potential time records sampled at 1 or $2 \mathrm{~Hz}$ for tests on dummy cells, excluding time records with a range greater than $5 \mathrm{mV}$ (DC levels offset for clarity): (A) RR1, $100 \Omega$ ( $N=1025$ to 16384$)$; (B) RR3, $100 \Omega(N=2048$ to 16500$)$; (C) RR1, $1 \mathrm{M} \Omega(N=1025$ to 16384); (D) RR3, $1 \mathrm{M} \Omega(N=2048$ to 16500$)$.

Fig. 3. Current time records sampled at 1 or $2 \mathrm{~Hz}$ for tests on dummy cells (DC levels offset for clarity): (A) RR1, $100 \Omega$ ( $N=1025$ to 16384$)$; (B) RR3, $100 \Omega(N=2048$ to 16500$)$; (C) $\mathrm{RR} 1,1 \mathrm{M} \Omega(N=1025$ to 16384$)$; (D) RR3, $1 \mathrm{M} \Omega(N=2048$ to 16500$)$.

Fig. 4. Potential power spectra for tests on dummy cells $(M=512)$. Dashed line: PSD of thermal noise: (A) RR1, $100 \Omega$; (B) RR3, $100 \Omega$; (C) RR1, 1 M $\Omega$; (D) RR3, $1 \mathrm{M} \Omega$.

Fig. 5. Current power spectra for tests on dummy cells $(M=512)$. Dashed line: PSD of thermal noise: (A) RR1, $100 \Omega$; (B) RR3, $100 \Omega$; (C) RR1, $1 \mathrm{M} \Omega$; (D) RR3, $1 \mathrm{M} \Omega$.

Fig. 6. Illustration of the effect of mains frequency noise and the role of anti-aliasing filters. Dashed line: PSD of thermal noise: (A) Potential time record sampled at $1 \mathrm{kHz}$ in RR1 $(\mathrm{R}=1 \mathrm{M} \Omega)$; (B) Right: PSD of the signal in (A), showing peaks at $50 \mathrm{~Hz}$ and harmonics; left: PSD measured at $f_{s}=1 \mathrm{~Hz}$ without mains interference; (C) Potential PSD for signals of $\mathrm{R}=10 \mathrm{k} \Omega$ in RR1, sampled at 1,20 , and $100 \mathrm{~Hz}$ showing peaks at $4.5 \mathrm{~Hz}$ and harmonics; (D) Potential PSD for R = $1 \mathrm{M} \Omega$ in RR1, sampled at $100 \mathrm{~Hz}$, showing the effect of an antialiasing filter with too low cut-off frequency.

Fig. 7. Illustration of the effect of quantisation noise: (A) Potential time record of $100 \Omega$ dummy cell in RR1 showing quantisation with a step size of $45 \mu \mathrm{V}$; (B) Corresponding power spectrum.

Fig. 8. Summary potential time records (mean value not removed) for Part 2 section (a): (A) RR2; (B) RR3.

Fig. 9. Summary potential time records sampled at 1 or $2 \mathrm{~Hz}$ for Part 2 (DC levels offset for clarity): (A) Section (a), RR2; (B) Section (a), RR3; (C) Section (b), RR2; (D) Section (b), RR3.

Fig. 10. Summary current time records sampled at 1 or $2 \mathrm{~Hz}$ for Part 2 (DC levels offset for clarity): (A) Section (a), RR2; (B) Section (a), RR3; (C) Section (b), RR2; (D) Section (b), RR3. 
Fig. 11. Potential and current time records for Part 2, section (a) for a system that appears to apply a regular autozero operation: (A) Potential time record sampled at $10.2 \mathrm{~Hz}$; (B) Corresponding current time record.

Fig. 12. Summary potential power spectra for Part $2(M=128)$ : (A) Section (a), RR2; (B) Section (a), RR3; (C) Section (b), RR2; (D) Section (b), RR3.

Fig. 13. Summary current power spectra for Part $2(M=128)$ : (A) Section (a), RR2; (B) Section (a), RR3; (C) Section (b), RR2; (D) Section (b), RR3. 


\section{Figures}

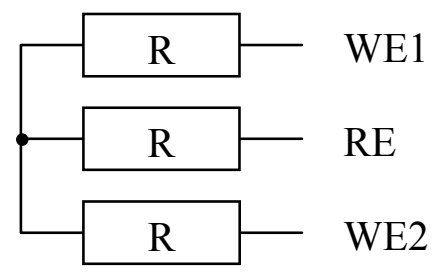

Fig. 1. Dummy cell for Part 1 (all resistors equal value).
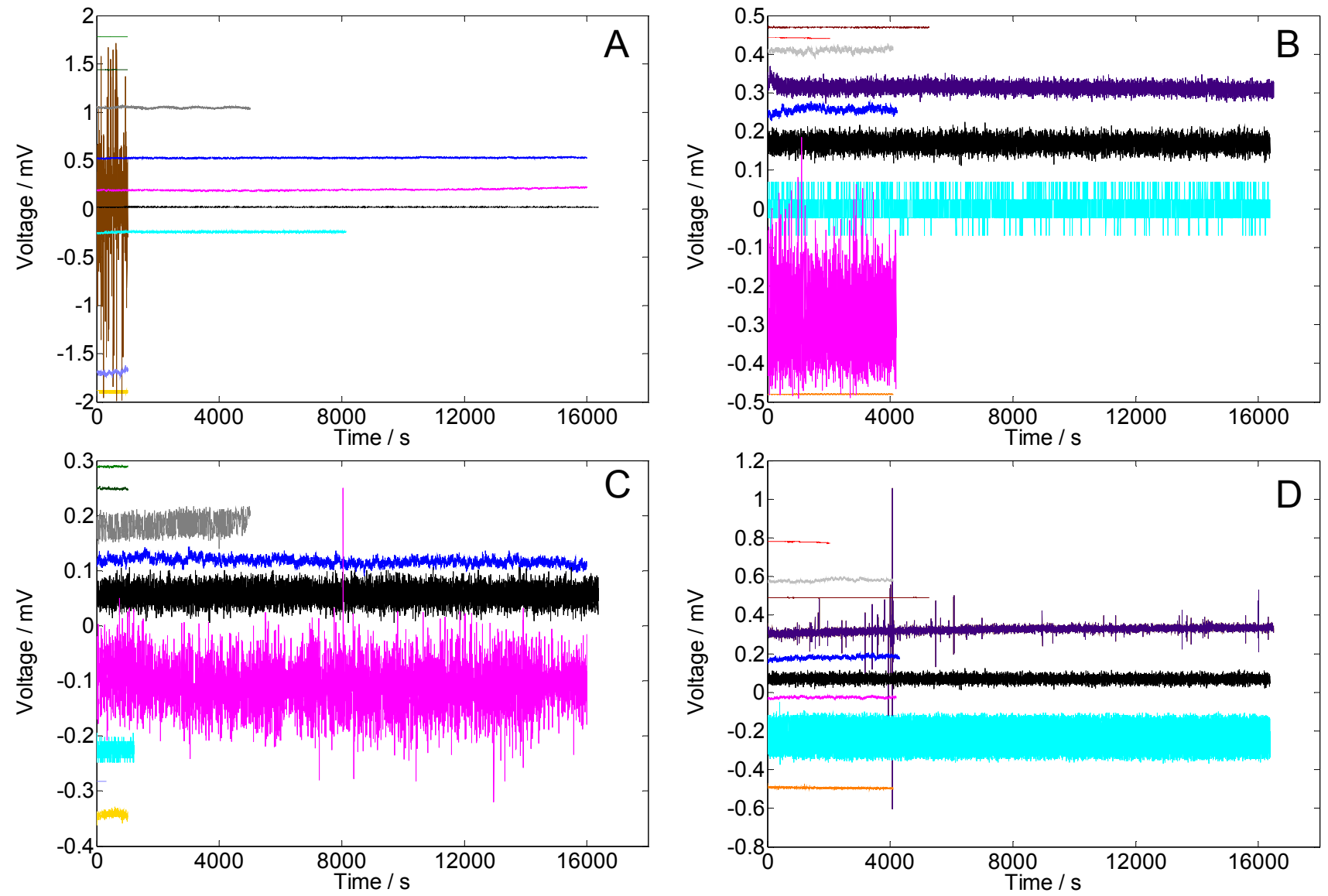

Fig. 2. Potential time records sampled at 1 or $2 \mathrm{~Hz}$ for tests on dummy cells, excluding time records with a range greater than $5 \mathrm{mV}$ (DC levels offset for clarity): (A) RR1, $100 \Omega$ $(N=1025$ to 16384$)$; (B) RR3, $100 \Omega(N=2048$ to 16500$)$; (C) RR1, $1 \mathrm{M} \Omega(N=1025$ to 16384); (D) RR3, $1 \mathrm{M} \Omega(N=2048$ to 16500$)$. 

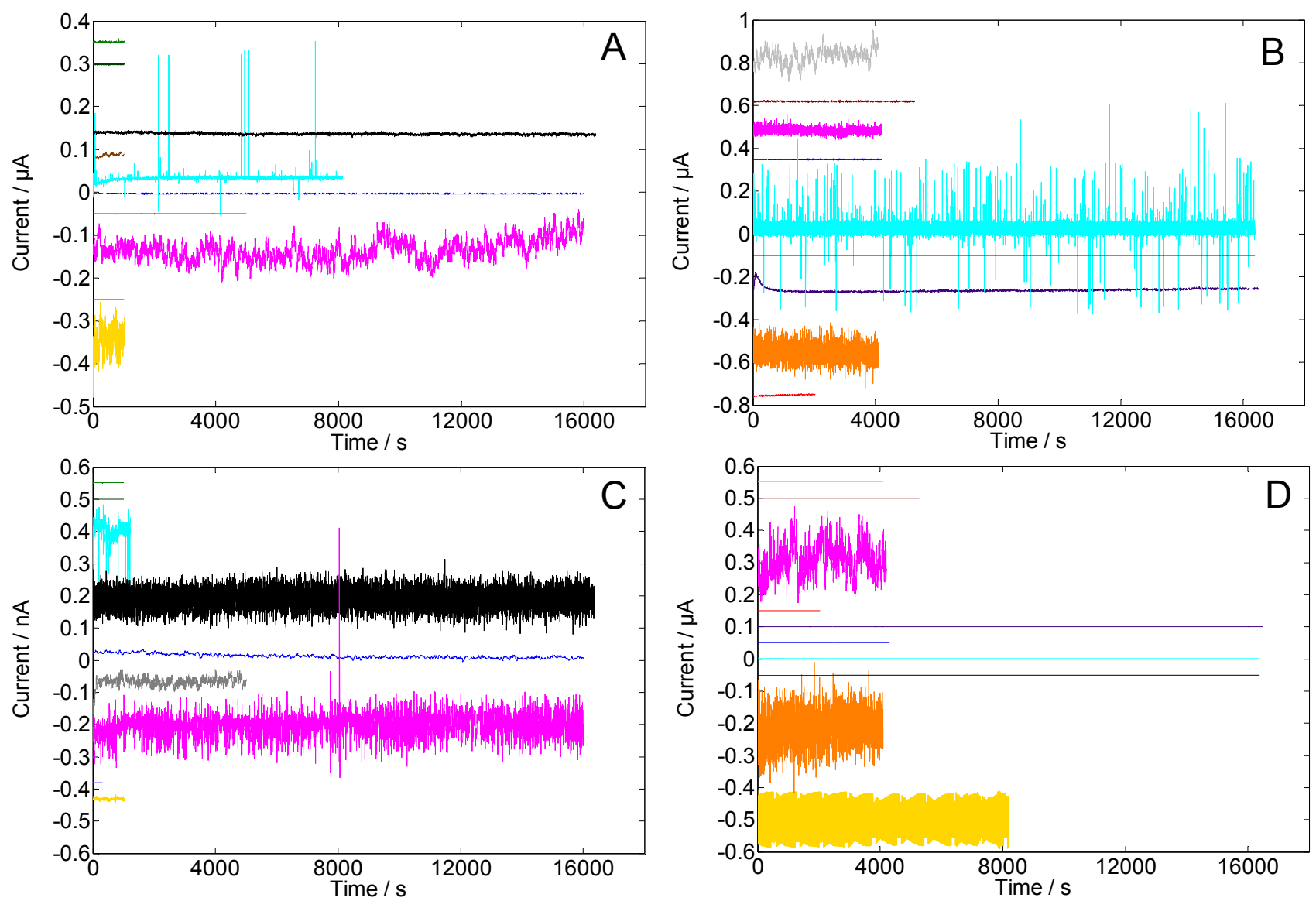

Fig. 3. Current time records sampled at 1 or $2 \mathrm{~Hz}$ for tests on dummy cells (DC levels offset for clarity): (A) RR1, $100 \Omega(N=1025$ to 16384$)$; (B) RR3, $100 \Omega(N=2048$ to 16500$)$; (C) RR1, $1 \mathrm{M} \Omega(N=1025$ to 16384$)$; (D) RR3, $1 \mathrm{M} \Omega(N=2048$ to 16500$)$. 

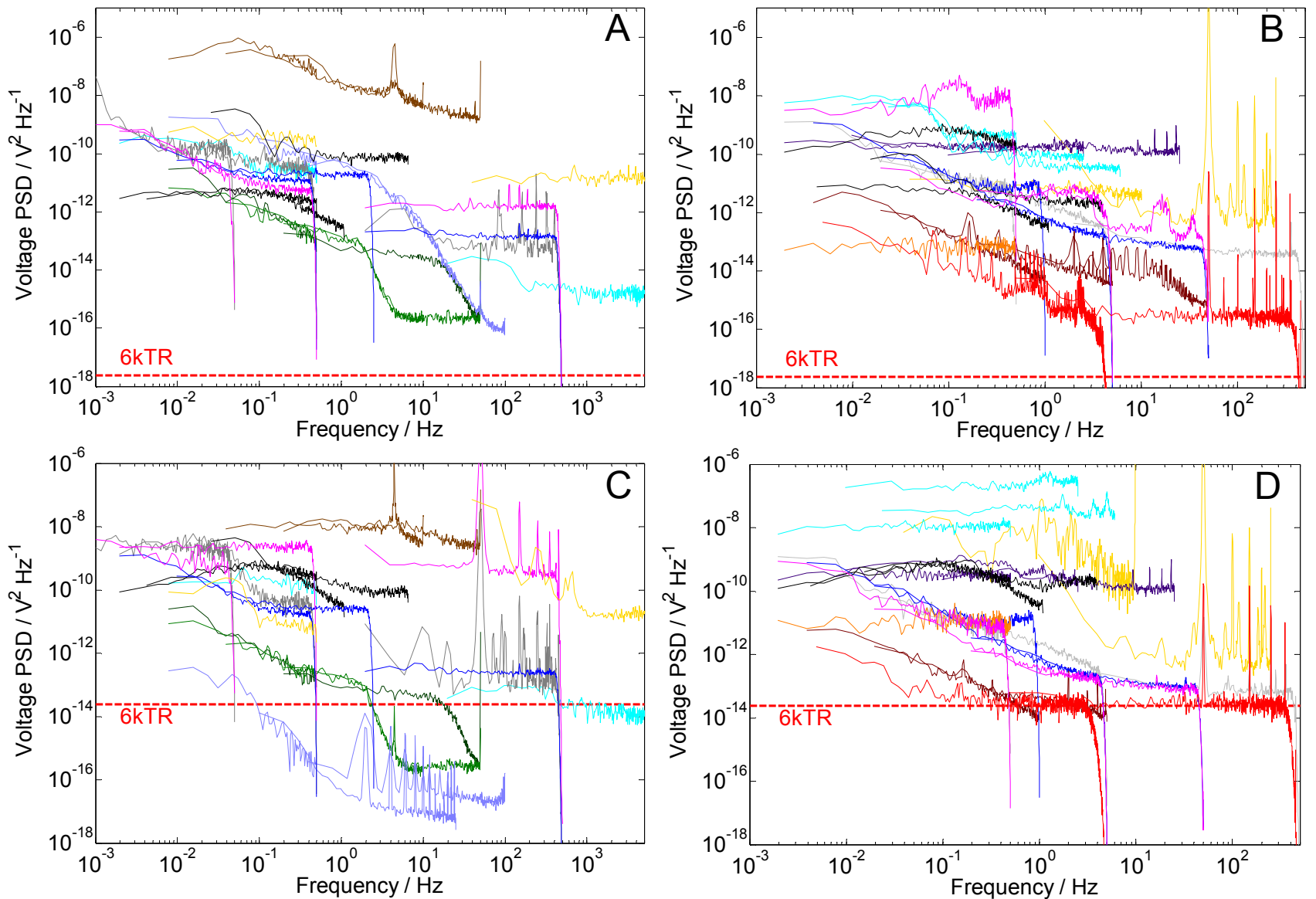

Fig. 4. Potential power spectra for tests on dummy cells $(M=512)$. Dashed line: PSD of thermal noise: (A) RR1, $100 \Omega$; (B) RR3, $100 \Omega$; (C) RR1, $1 \mathrm{M} \Omega$; (D) RR3, $1 \mathrm{M} \Omega$. 

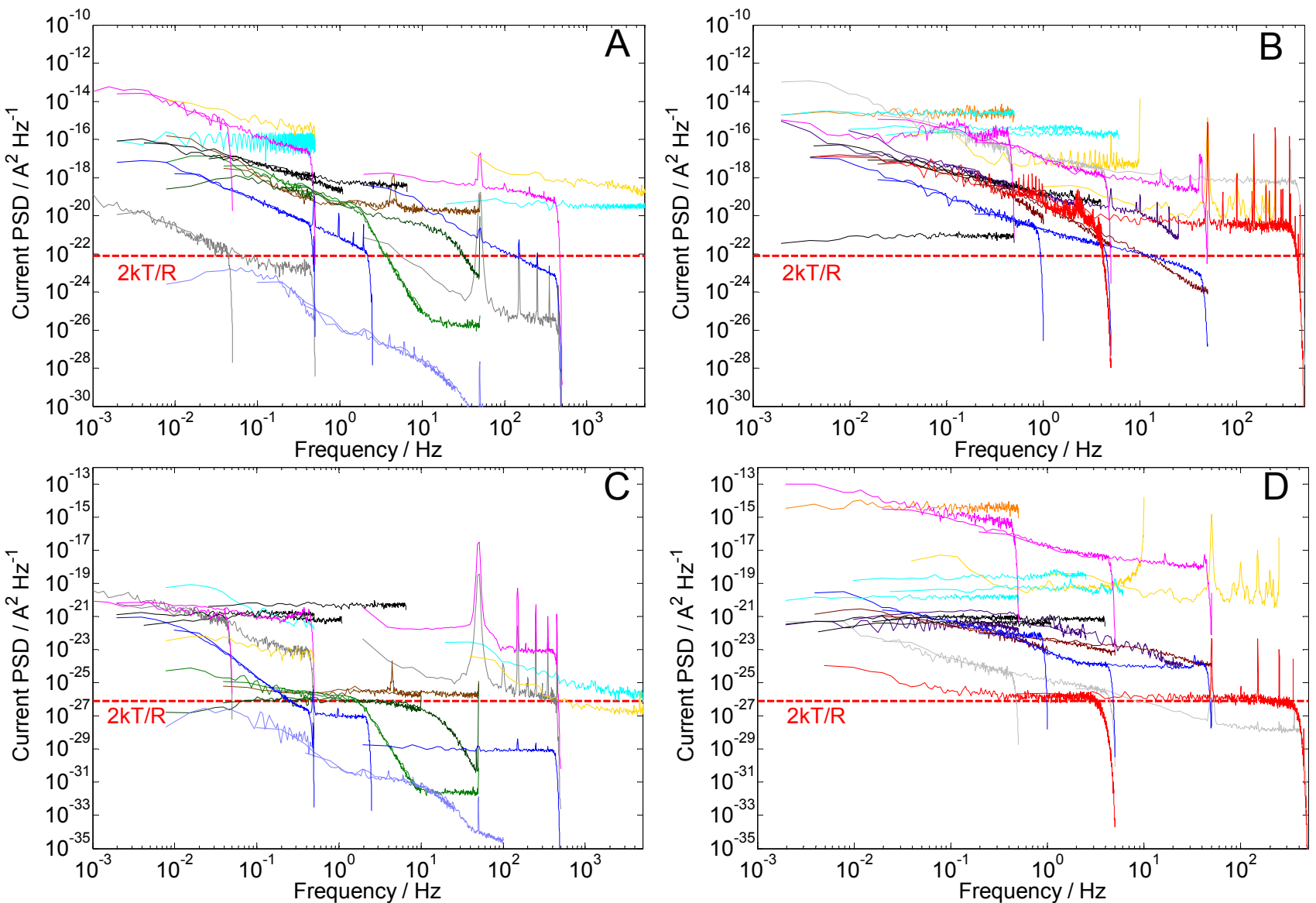

Fig. 5. Current power spectra for tests on dummy cells $(M=512)$. Dashed line: PSD of thermal noise: (A) RR1, $100 \Omega$; (B) RR3, $100 \Omega$; (C) RR1, 1 M $\Omega$; (D) RR3, $1 \mathrm{M} \Omega$. 

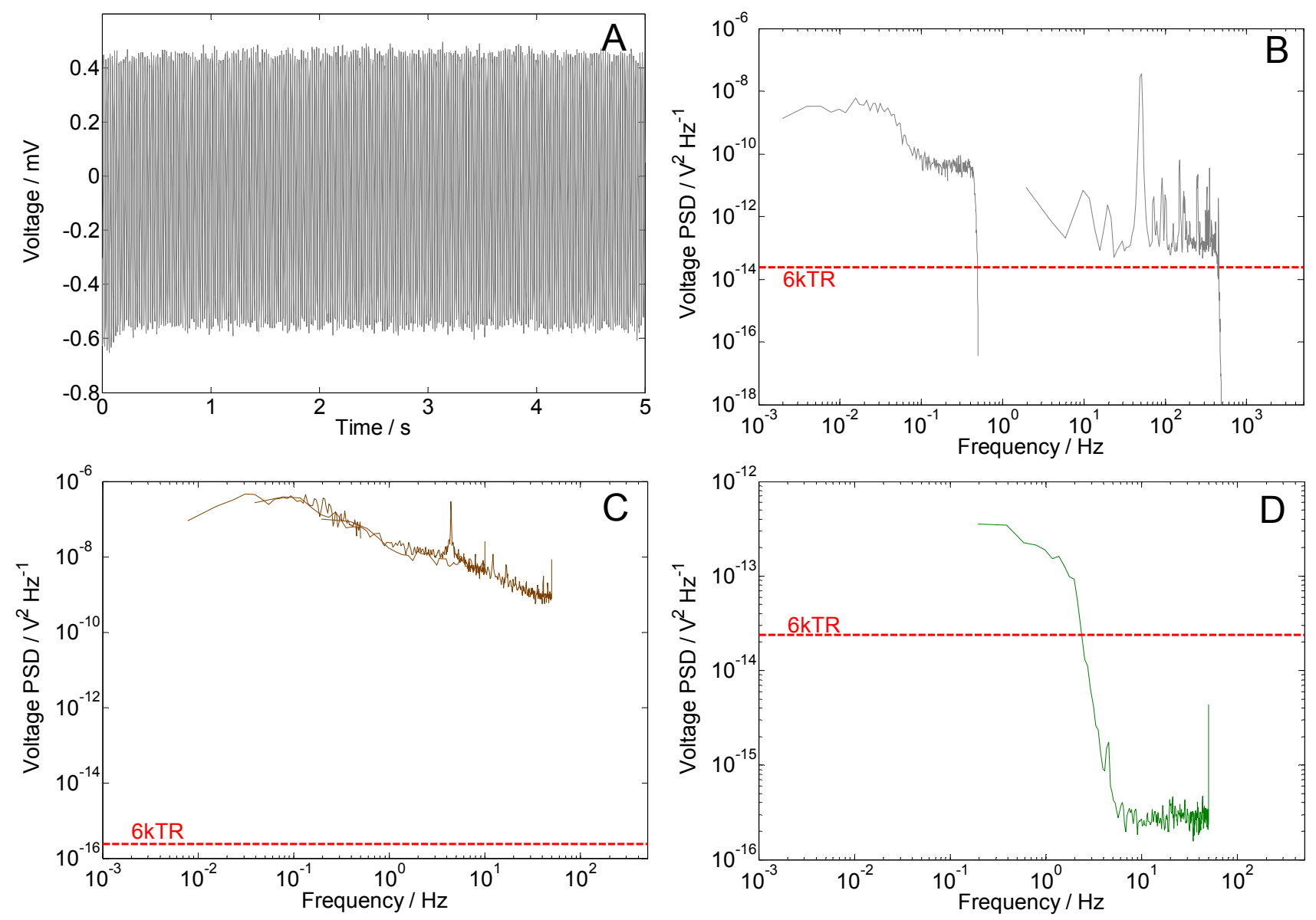

Fig. 6. Illustration of the effect of mains frequency noise and the role of anti-aliasing filters.

Dashed line: PSD of thermal noise: (A) Potential time record sampled at $1 \mathrm{kHz}$ in RR1 $(\mathrm{R}=1 \mathrm{M} \Omega)$; (B) Right: PSD of the signal in (A), showing peaks at $50 \mathrm{~Hz}$ and harmonics; left: PSD measured at $f_{s}=1 \mathrm{~Hz}$ without mains interference; (C) Potential PSD for signals of $\mathrm{R}=10 \mathrm{k} \Omega$ in $\mathrm{RR} 1$, sampled at 1,20 , and $100 \mathrm{~Hz}$ showing peaks at $4.5 \mathrm{~Hz}$ and harmonics; (D) Potential PSD for R $=1 \mathrm{M} \Omega$ in RR1, sampled at $100 \mathrm{~Hz}$, showing the effect of an antialiasing filter with too low cut-off frequency. 

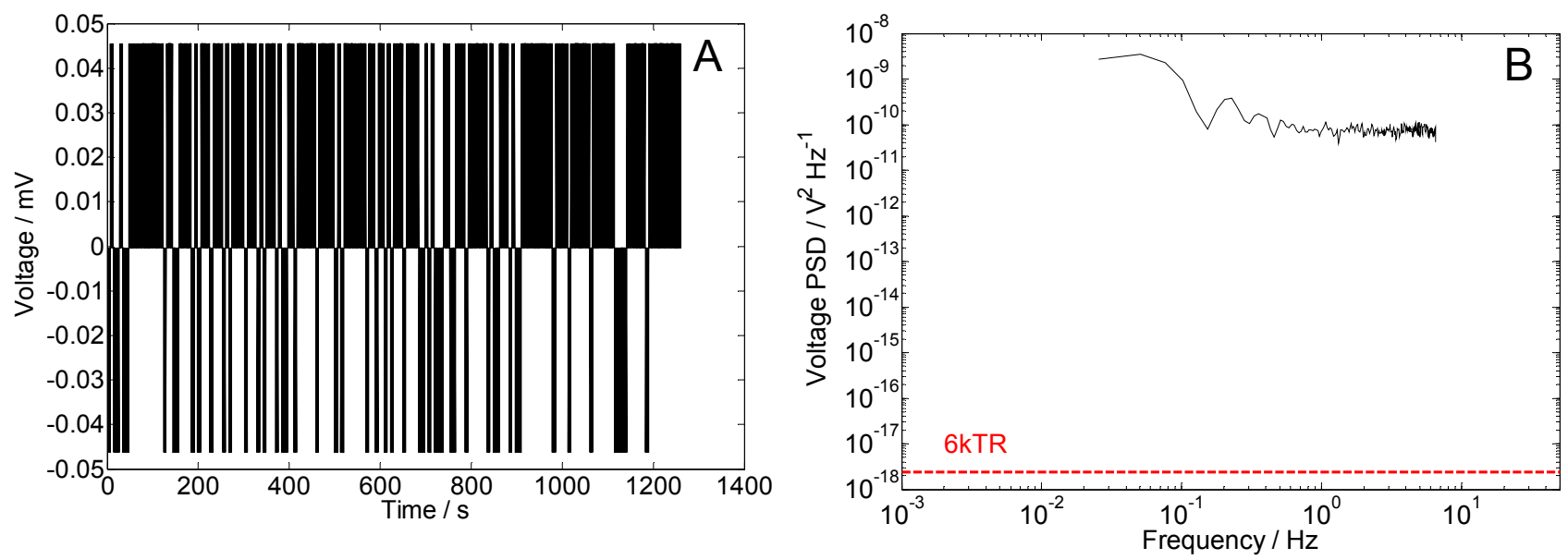

Fig. 7. Illustration of the effect of quantisation noise: (A) Potential time record of $100 \Omega$ dummy cell in RR1 showing quantisation with a step size of $45 \mu \mathrm{V}$; (B) Corresponding power spectrum.
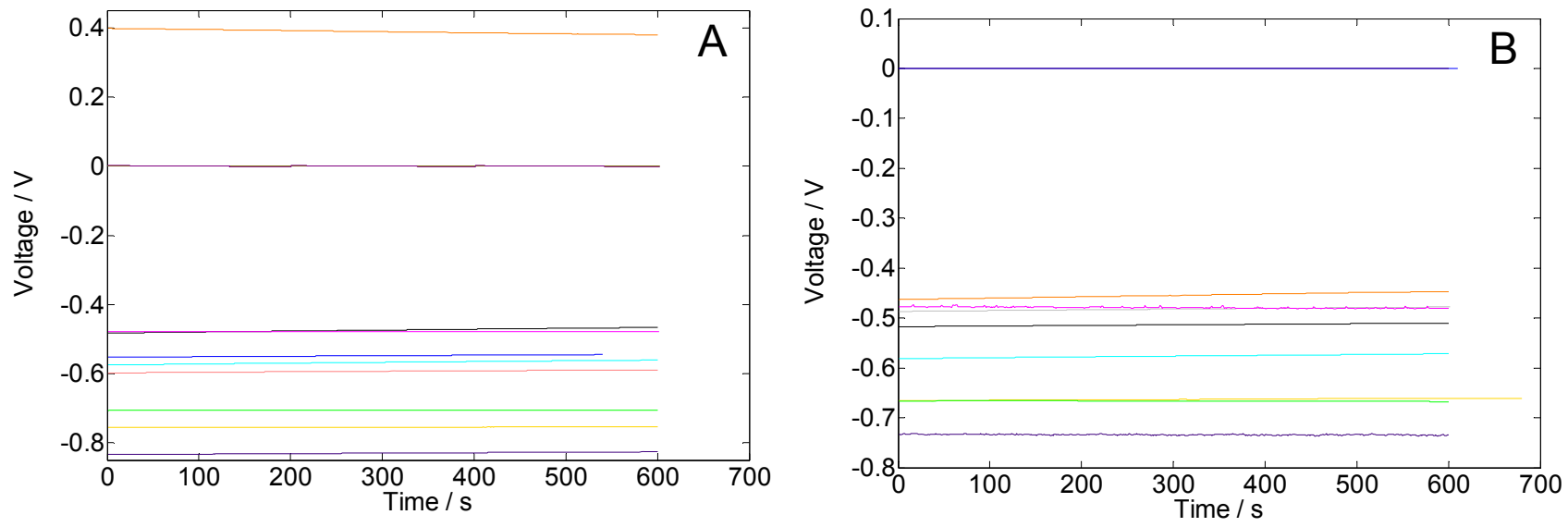

Fig. 8. Summary potential time records (mean value not removed) for Part 2 section (a): (A) RR2; (B) RR3. 

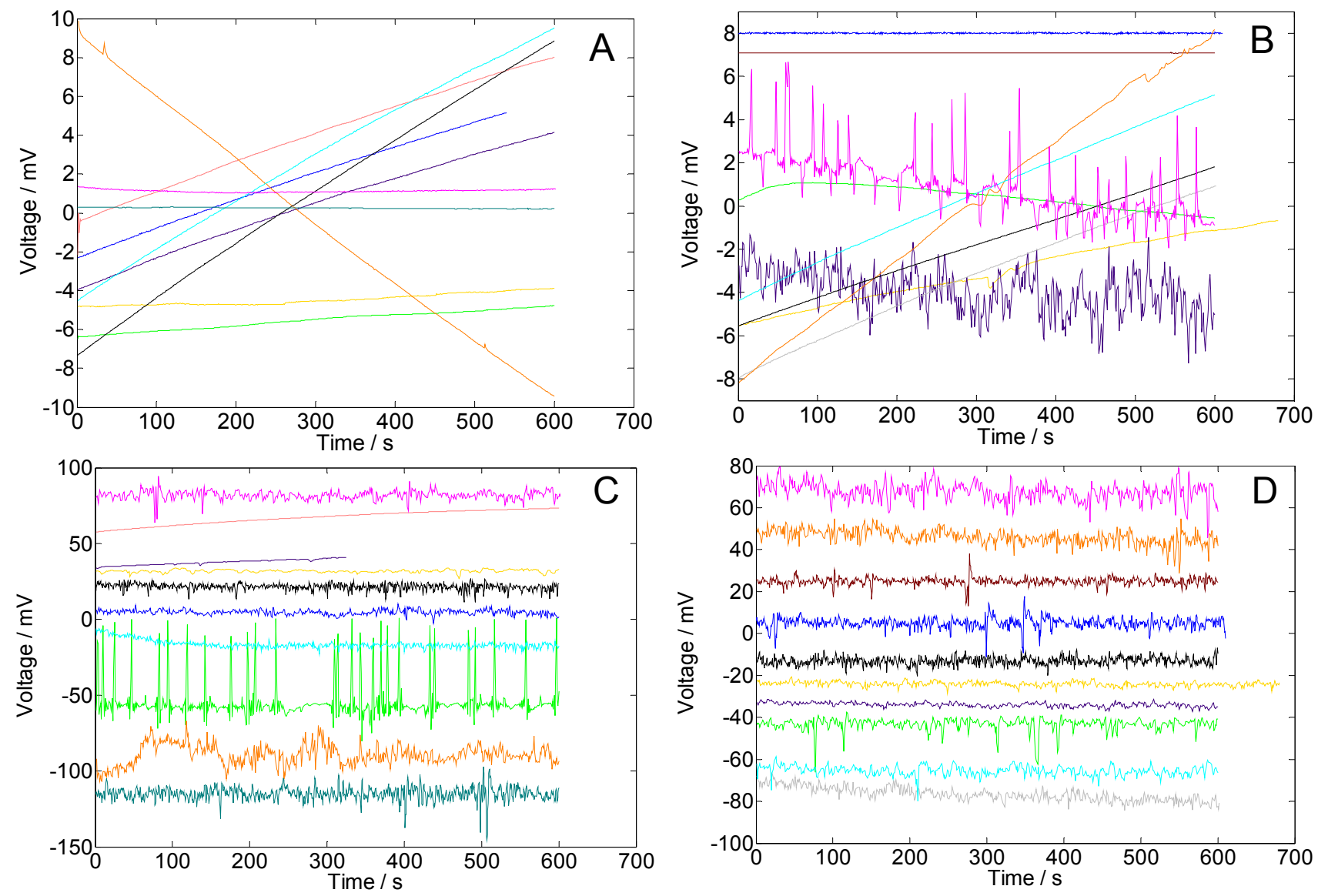

Fig. 9. Summary potential time records sampled at $1 \mathrm{or} 2 \mathrm{~Hz}$ for Part 2 (DC levels offset for clarity): (A) Section (a), RR2; (B) Section (a), RR3; (C) Section (b), RR2; (D) Section (b), RR3. 

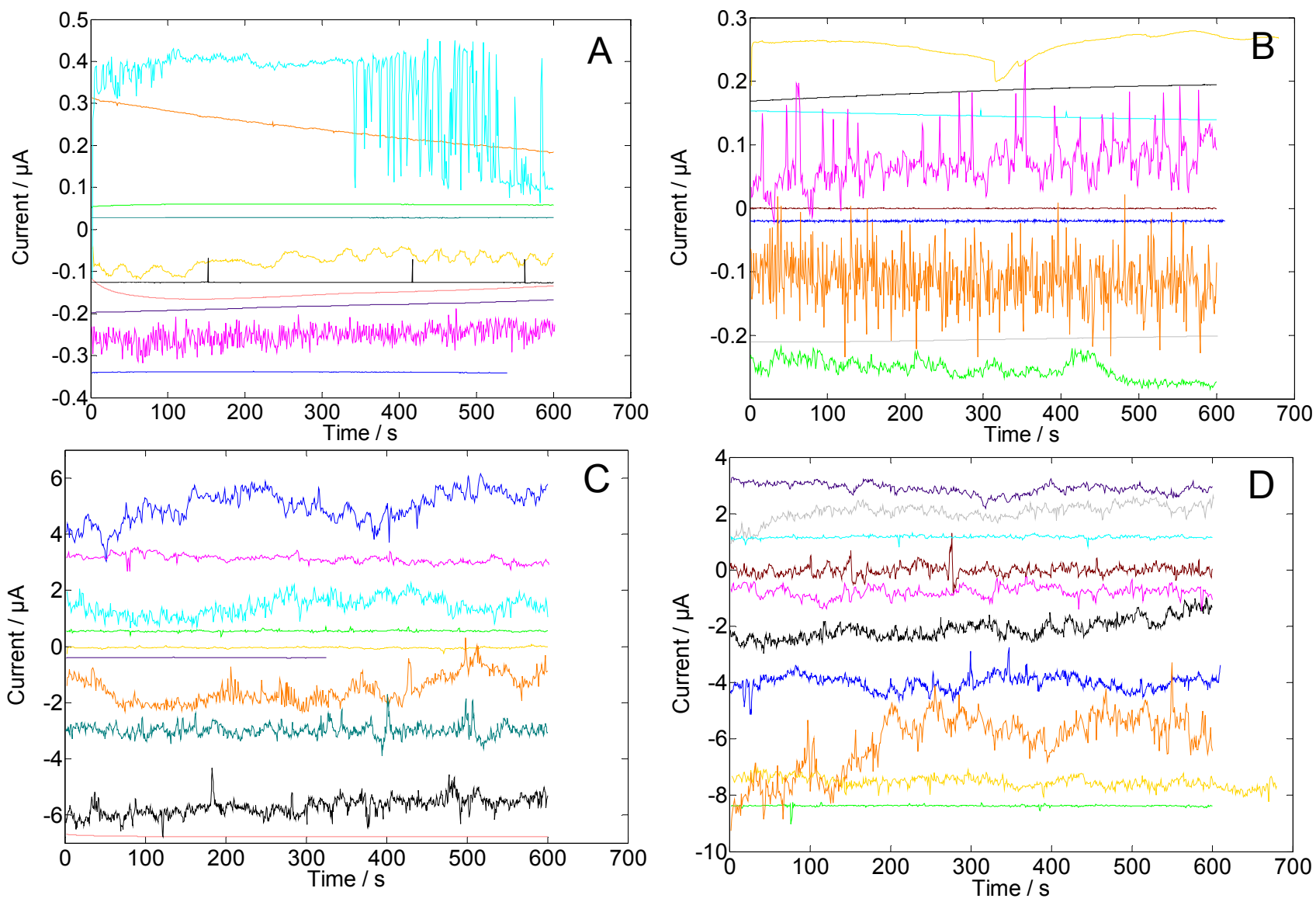

Fig. 10. Summary current time records sampled at 1 or $2 \mathrm{~Hz}$ for Part 2 (DC levels offset for clarity): (A) Section (a), RR2; (B) Section (a), RR3; (C) Section (b), RR2; (D) Section (b), RR3. 

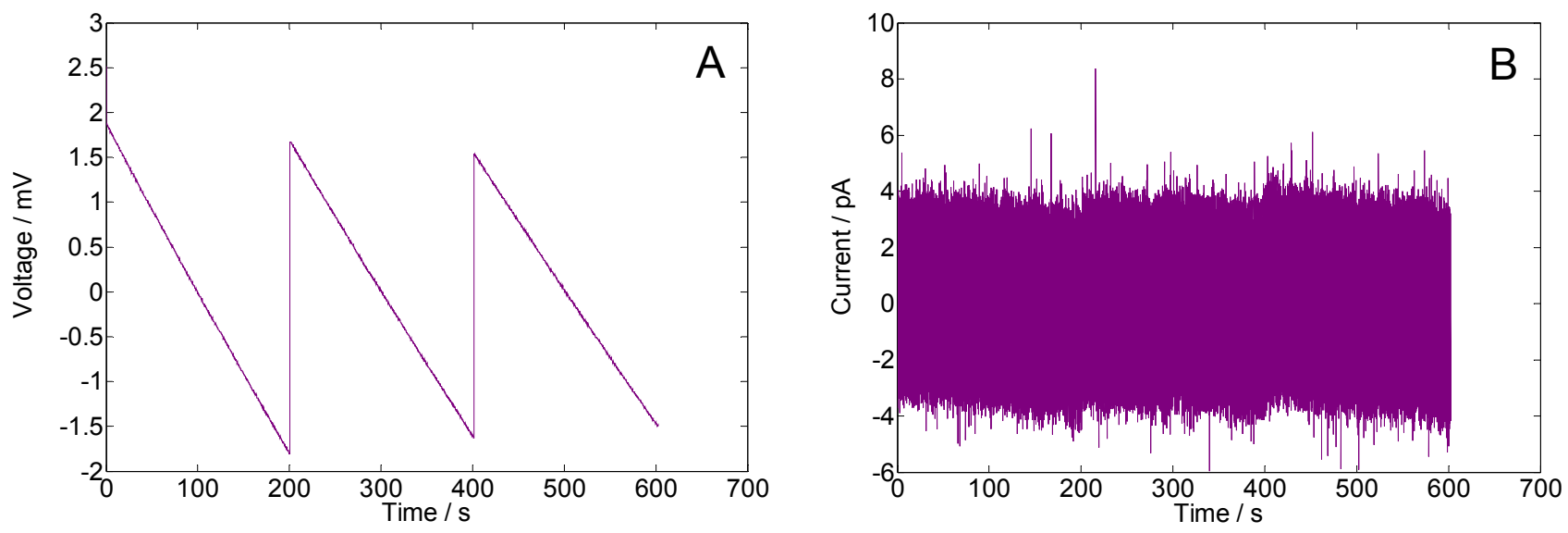

Fig. 11. Potential and current time records for Part 2, section (a) for a system that appears to apply a regular autozero operation: (A) Potential time record sampled at $10.2 \mathrm{~Hz}$; (B) Corresponding current time record. 

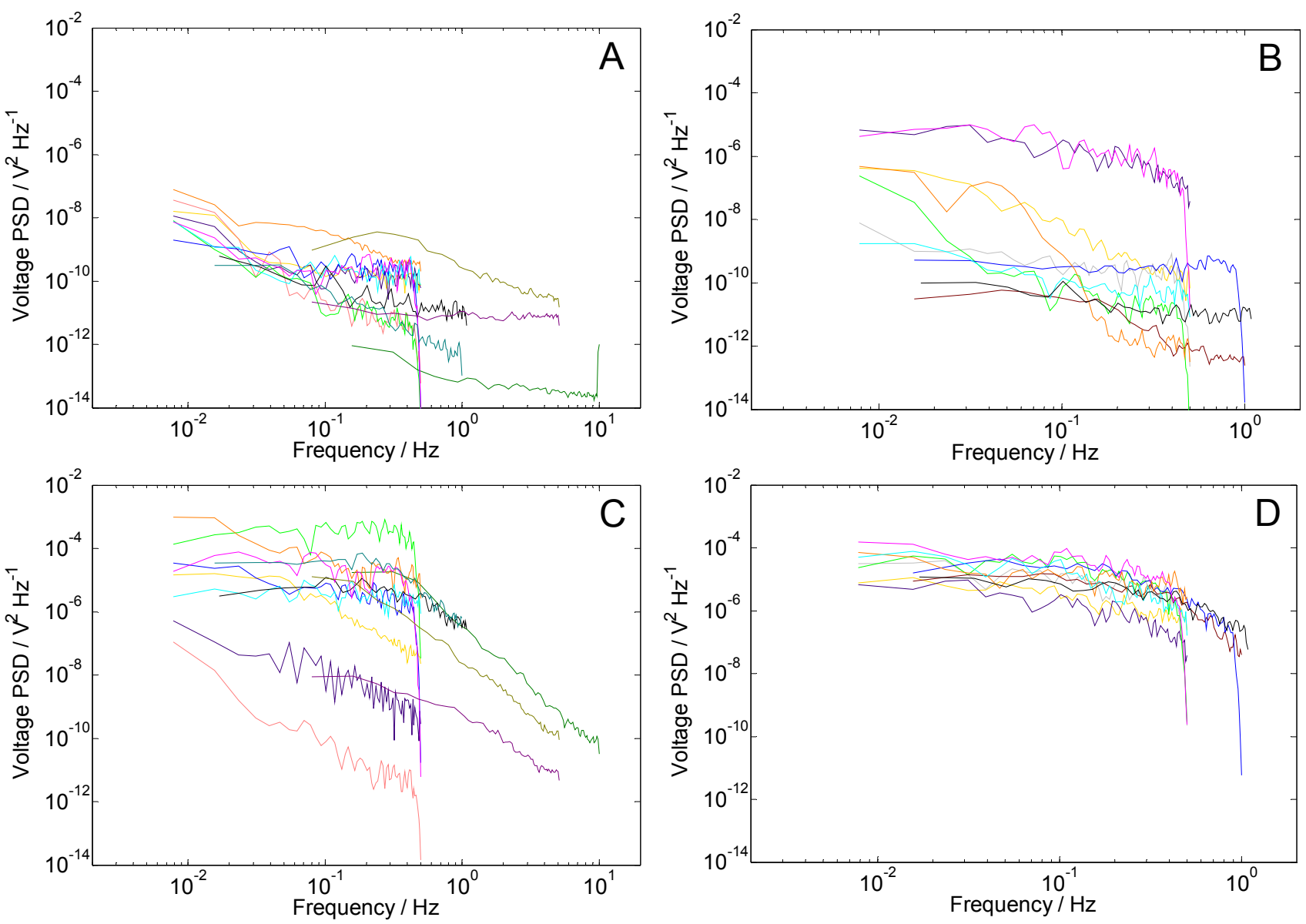

Fig. 12. Summary potential power spectra for Part $2(M=128)$ : (A) Section (a), RR2; (B) Section (a), RR3; (C) Section (b), RR2; (D) Section (b), RR3. 

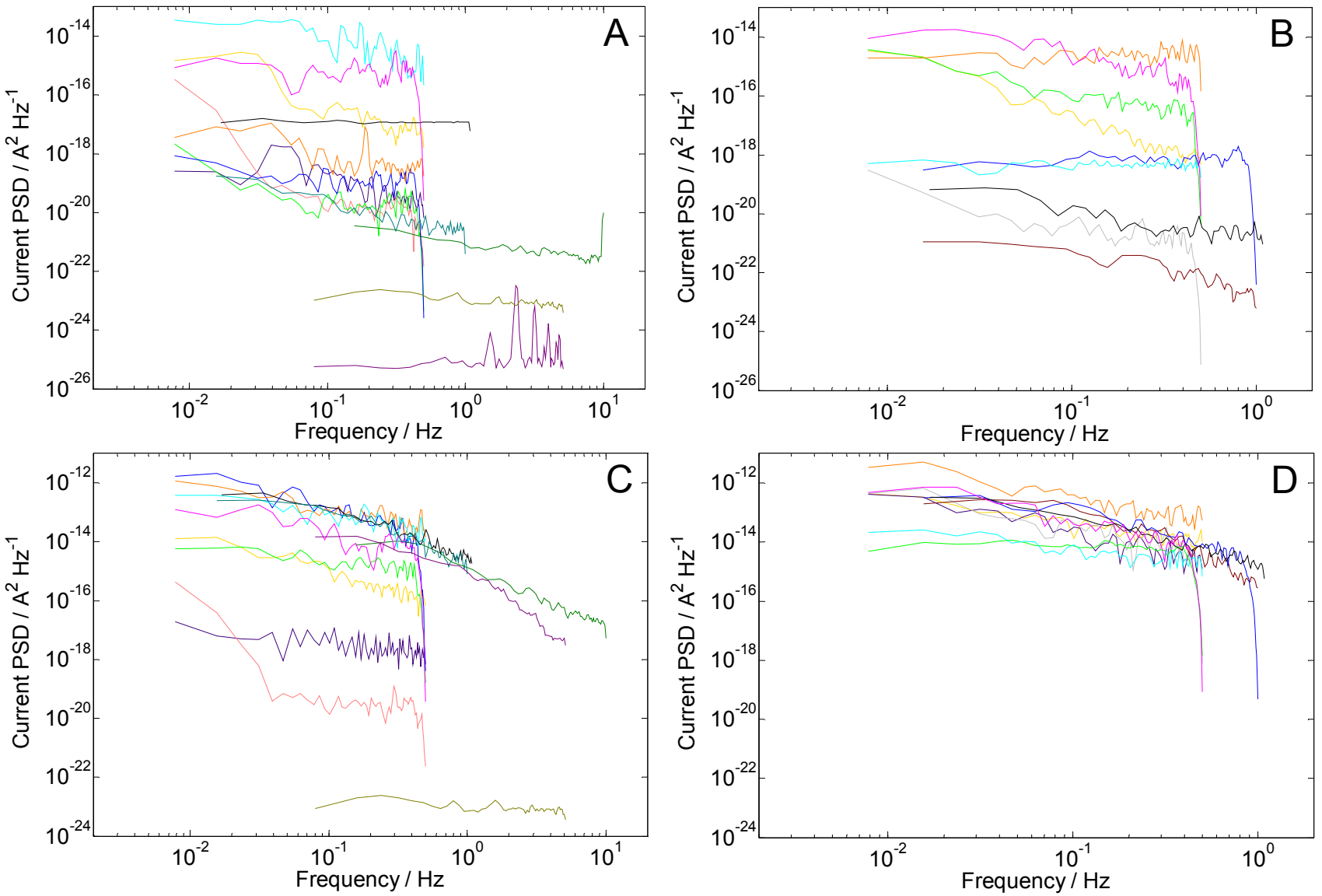

Fig. 13. Summary current power spectra for Part $2(M=128)$ : (A) Section (a), RR2; (B) Section (a), RR3; (C) Section (b), RR2; (D) Section (b), RR3. 